\title{
Adaptation and Inhibition Control Pathological Synchronization in a Model of Focal Epileptic Seizure
}

\author{
(D)Atoly Buchin, ${ }^{1 *}$ Cliff C. Kerr, ${ }^{2}$ ' Gilles Huberfeld, ${ }^{3,4}$ Richard Miles, ${ }^{5}$ and ${ }^{\circledR}$ Boris Gutkin ${ }^{6,7}$
}

https://doi.org/10.1523/ENEURO.0019-18.2018

\begin{abstract}
${ }^{1}$ University of Washington, Department of Physiology and Biophysics (United States, Seattle), 1959 NE Pacific St, 98195, '2University of Sydney, School of Physics (Australia, Sydney), Physics Rd, NSW 2006, '35orbonne UniversitéUPMC, Pitié-Salpêtrière Hô, Neurophysiology Department (France, Paris), 47-83 Boulevard de l'Hôpital, 75013, ${ }^{4}$ Institut national de la santé et de la recherche médicale Unit 1129 "Infantile Epilepsies and Brain Plasticity", Paris Descartes University, Sorbonne Paris Cité University group, (France, Paris), 149 rue de Sévres $75015,{ }^{5}$ Brain and Spine Institute, Cortex and Epilepsie Group (France, Paris), 47 Boulevard Hôpital, 75013, ${ }^{6}$ Paris Sciences \& Lettres Research University, Laboratoire des Neurosciences Cognitives, Group for Neural Theory (France, Paris), 29, rue d'Ulm, 75005 France, and ${ }^{7}$ National Research University Higher School of Economics, Center for Cognition and Decision Making (Russia, Moscow), 20 Myasnitskaya, 109316
\end{abstract}

\section{Visual Abstract}

\section{Resting state}
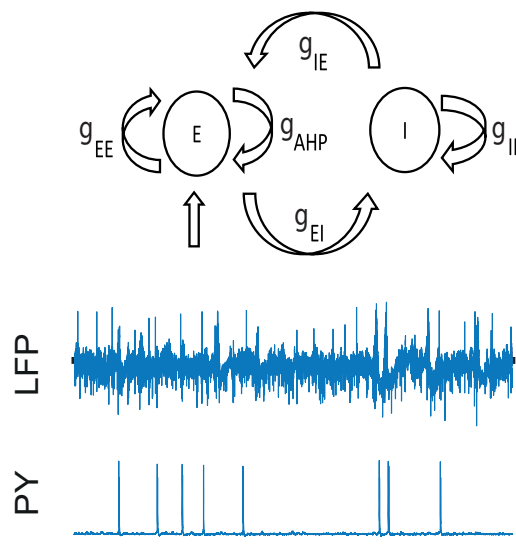

Pre-ictal discharges
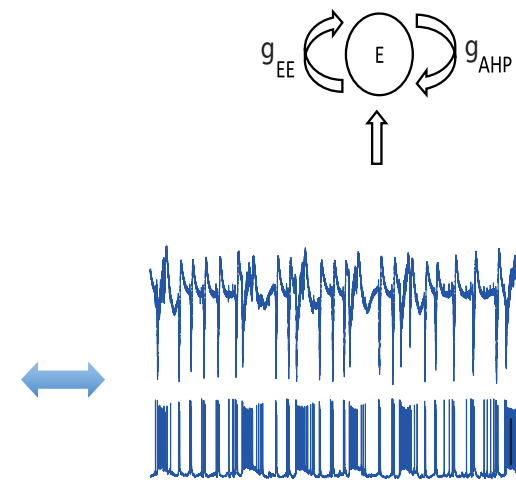

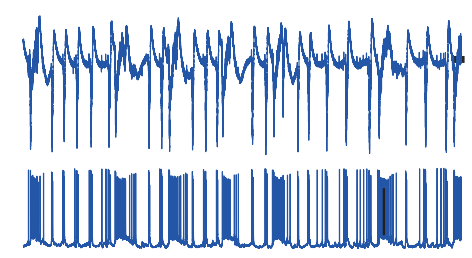

Seizure
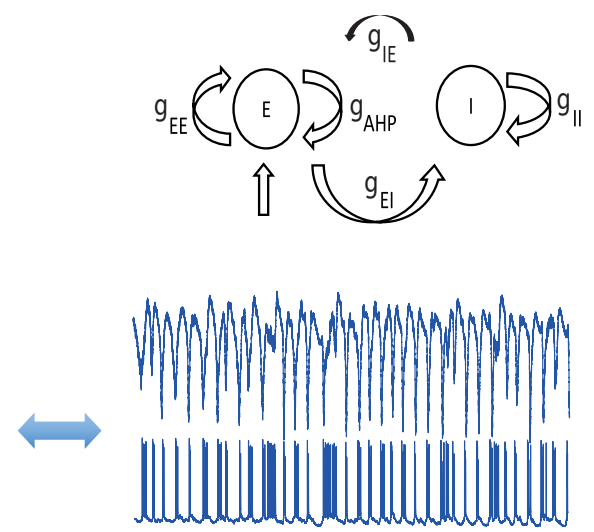

\section{Significance Statement}

Epileptic seizures are commonly thought to arise from a pathology of inhibition in the brain circuits. Theoretical models aiming to explain epileptic oscillations usually describe the neural activity solely in terms of inhibition and excitation. Single neuron adaptation properties are usually assumed to have only a limited contribution to seizure dynamics. To explore this issue, we developed a novel neural mass model with adaption in the excitatory population. By including adaptation together with inhibition in this model, we were able to account for several experimentally observed properties of seizures, resting state dynamics, and pre-ictal oscillations, leading to improved understanding of epileptic seizures. 
Pharmacoresistant epilepsy is a common neurological disorder in which increased neuronal intrinsic excitability and synaptic excitation lead to pathologically synchronous behavior in the brain. In the majority of experimental and theoretical epilepsy models, epilepsy is associated with reduced inhibition in the pathological neural circuits, yet effects of intrinsic excitability are usually not explicitly analyzed. Here we present a novel neural mass model that includes intrinsic excitability in the form of spike-frequency adaptation in the excitatory population. We validated our model using local field potential (LFP) data recorded from human hippocampal/subicular slices. We found that synaptic conductances and slow adaptation in the excitatory population both play essential roles for generating seizures and pre-ictal oscillations. Using bifurcation analysis, we found that transitions towards seizure and back to the resting state take place via Andronov-Hopf bifurcations. These simulations therefore suggest that single neuron adaptation as well as synaptic inhibition are responsible for orchestrating seizure dynamics and transition towards the epileptic state.

Key words: adaptation; AHP current; neural mass model; synaptic noise; temporal lobe epilepsy

\section{Introduction}

Epilepsy is the fourth most common neurologic disorder, and is responsible for a greater total global burden of disease than any neurologic conditions except for stroke and migraine (Beghi et al., 2005; Rothstein et al., 2005; Chin and Vora, 2014). Epileptic seizures are characterized by the increased excitability/excitation in the brain's recurrently coupled neuronal networks (Lytton, 2008). Typically, experimental seizure models assume that seizures occur due to decreased inhibition (Karnup and Stelzer, 1999; Sivakumaran et al., 2015) or increased excitation in the neural networks (Ursino and la Cara, 2006; Hall and Kuhlmann, 2013).

There is also evidence that interneurons increase their firing at seizure initiation (Lillis et al., 2012) and are active during the time course of the epileptic activity (Ziburkus et al., 2006), suggesting that the activity of interneurons contributes importantly to aspects of seizure dynamics. The activity-dependent interplay between the pyramidal cells and interneurons could play an essential role for seizure generation mechanisms (Krishnan and Bazhenov, 2011; Naze et al., 2015; Buchin et al., 2016b). In neural mass models, neuron populations are often treated as rate units lacking intrinsic adaptation (Touboul et al., 2011). The dy-

Received January 10, 2018; accepted June 7, 2018; First published September 13,2018

The authors declare no competing financial interests.

Author contributions: A.B., C.C.K., G.H., R.M., and B.G. designed research; A.B. and G.H. performed research; A.B. and C.C.K. analyzed data; A.B., C.C.K., G.H., R.M., and B.G. wrote the paper.

This work was supported by the Swartz Foundation, FRM FDT20140930942, ANR-10-LABX-0087 IEC, and ANR-10-IDEX-0001-02 PSL grants. B.G. was supported by funding from the RF (Russian Federation) Program 5-100 to the National Research University Higher School of Economics. C.C.K. was supported by the Australian Research Council (ARC) Discovery Early Career Researcher Award DE140101375.

${ }^{*}$ A. Buchin's present address: Allen Institute for Brain Science, Modelling Analysis and Theory, 615 Westlake Avenue North, Seattle, WA 98109.

Acknowledgements: We thank Anton Chizhov for useful comments and criticism of our work. Initial ideas were developed during the Advanced Course in Computational Neuroscience in Będlevo, Poland (http://www.neuroinf.pl/ accn).

Correspondence should be addressed to Anatoly Buchin at the above address, E-mail: anat.buchin@gmail.com.

https://doi.org/10.1523/ENEURO.0019-18.2018

Copyright (C) 2018 Buchin et al.

This is an open-access article distributed under the terms of the Creative Commons Attribution 4.0 International license, which permits unrestricted use, distribution and reproduction in any medium provided that the original work is properly attributed. namic behavior of the neural populations is determined by the balance between excitation and inhibition. Despite the simplicity of these models, they can be successfully used to reproduce resting and interictal states as well as ictal discharges by producing time series comparable with macroscopic measurements such as electroencephalogram signals and field potentials (Demont-Guignard et al., 2009).

However, not all types of epileptic seizures can be explained by looking only at the balance between excitation and inhibition (Traub et al., 2005); intrinsic excitability changes on the single-neuron level also play an important role (Krishnan and Bazhenov, 2011). Studies on human subiculum tissue showed that the complete blockade of type A GABAergic neurotransmission (and thus inactivation of the effects of inhibitory population) precludes seizure emergence while, if applied after seizure initiation, it abolishes rather than enhances the seizure activity. These manipulations usually bring back the neural network in the slice toward pre-ictal events, which have substantially different frequency content than seizure activity (Huberfeld et al., 2011), and which in this case fail to trigger ictal events. In human epileptic tissues, including peritumoral neocortex (Pallud et al., 2014), interictal discharges are generated spontaneously. These events are triggered by interneurons which depolarize pyramidal cells with impaired chloride regulation, leading to depolarizing effects of GABA. Once activated, pyramidal cells excite other cells via AMPA-mediated glutamatergic transmission. In these tissues, seizures can be produced by increasing local excitability using modified bathing media. The transition to seizures is characterized by the emergence of specific pre-ictal events initiated by pyramidal cells which synchronize local neurons by AMPA synapses. These pre-ictal events cluster before seizure initiation which requires functional AMPA, NMDA as well as GABA $A_{A}$ signals. The conventional neural mass models are unable to explain these pre-ictal oscillations because they require the excitatory population to generate periodic oscillations in the absence of inhibition. The second motivation for incorporating intrinsic excitability into neural mass models is that in epileptogenic areas, such as human subiculum, there is a substantial proportion of neurons with non-trivial intrinsic properties such as spike-frequency adaptation (Jensen et al., 1994; Huberfeld et al., 2007). To take these properties into account, neural mass models need to be enriched by the addition of components such as slow potassium currents (Pinsky and Rinzel, 1994). 
In addition, seizures are typically accompanied by high potassium concentrations (Dietzel and Heinemann, 1986; Xiong and Stringer, 1999; Fröhlich and Bazhenov, 2006; Florence et al., 2009), which in turn activate calcium currents (Bazhenov et al., 2004; Fröhlich et al., 2008), which in turn affect spike-frequency adaptation and intrinsic bursting. These properties are likely to modulate the single neuron firing and thus further influence the neuronal dynamics. These findings motivate the development of neural mass models that can capture the intrinsic excitability in coupled neural populations.

In this work, we developed a novel neural mass model consisting of an inhibitory neural population and an adaptive excitatory neuronal population (Buchin and Chizhov, $2010 \mathrm{~b})$. We calibrated the parameters of the model to local field potential (LFP) data recorded in human subiculum slices during rest, seizure, and full disinhibition in pre-ictal condition. We then analyzed the model as calibrated to each of these three regimes. Our results emphasize the role of intrinsic excitability such as adaptation in the excitatory population, which help explain the transitions between rest, seizure, and full disinhibition states.

\section{Materials and Methods}

\section{Epileptic tissue}

Temporal lobe tissue blocks containing the hippocampus, subiculum, and part of the entorhinal cortex were obtained from 45 people of both sexes with pharmacoresistant medial temporal lobe epilepsies associated with hippocampal sclerosis (age, 18-52 years; seizures for 3-35 years) undergoing resection of the amygdala, the hippocampus, and the anterior parahippocampal gyrus. All of the individuals gave their written informed consent and the study was approved by the Comite Consultatif National d'Ethique.

\section{Tissue preparation}

The post-surgical tissue was transported in a cold, oxygenated solution containing $248 \mathrm{mM}$ D-sucrose, 26

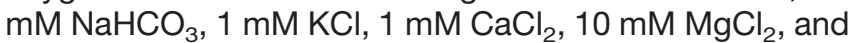
$10 \mathrm{mM}$ D-glucose, equilibrated with $5 \% \mathrm{CO}_{2}$ in $95 \% \mathrm{O}_{2}$. Hippocampal-subicular-entorhinal cortical slices or isolated subicular slices (400- $\mu \mathrm{m}$ thickness, $3 \times 12 \mathrm{~mm}$ length and width) were cut with a vibratome (HM650 V, Microm). They were maintained at $37^{\circ} \mathrm{C}$ and equilibrated with $5 \% \mathrm{CO}_{2}$ in $95 \% \mathrm{O}_{2}$ in an interface chamber perfused with a solution containing $124 \mathrm{mM} \mathrm{NaCl}, 26 \mathrm{mM} \mathrm{NaHCO}_{3}$, $4 \mathrm{mM} \mathrm{KCl}, 2 \mathrm{mM} \mathrm{MgCl} 2,2 \mathrm{mM} \mathrm{CaCl}$, and $10 \mathrm{mM}$ $\mathrm{D}$-glucose. Bicuculline or picrotoxin was used to block $\mathrm{GABA}_{\mathrm{A}}$ receptors. Ictal-like activity was induced by increasing the external $\mathrm{K}^{+}$concentration to $8 \mathrm{mM}$ and reducing the $\mathrm{Mg}^{2+}$ concentration to $0.25 \mathrm{mM}$ to increase the cellular excitability (similar to Huberfeld et al., 2011).

\section{Recordings}

Up to four tungsten electrodes etched to a tip diameter of $\sim 5 \mu \mathrm{m}$ were used for the extracellular recordings. The signals were amplified 1000 -fold and filtered to pass frequencies of $0.1 \mathrm{~Hz}$ to $10 \mathrm{kHz}$ (AM Systems, 1700). The extracellular signals were digitized at $10 \mathrm{kHz}$ with a 12-bit, 16-channel A-D converter (Digidata 1200A, Molecular Devices) and monitored and saved to a PC with Axoscope (Molecular Devices).

\section{Data analysis}

Records were analyzed using pCLAMP 10 software and scripts written in MATLAB 2016a. Power spectrum estimation was performed using fast Fourier transforms. The major frequencies of oscillations were computed via the multitaper method (Thomson, 1982).

\section{Simulations and analysis}

Neural population model simulations were performed in XPPAUT 8.0 using the direct Euler method of integration, with a time step of $0.05 \mathrm{~ms}$. Smaller time steps were tested and provided substantially similar results. In all simulations the initial conditions were systematically varied to check stability of numerical results. The data for the model was taken from one representative patient in the brain slice demonstrating resting state, seizure and preictal oscillations.

\section{Software accessibility}

The model code is available on GitHub (https://github.com/ abuchin/El-with-adaptation). Bifurcation analysis was performed in the XPP AUTO package (http://www.math.pitt.edu/ bard/xpp/xpp.html). All code is also available as Extended Data 1.

\section{Neural mass model}

In the model we considered interacting excitatory and inhibitory neural populations coupled by AMPA and $\mathrm{GABA}_{\mathrm{A}}$ synapses. All model parameters and variables are presented in Tables 1, 2. Each population was characterized by the average membrane potential of a population of leaky integrate-and-fire (LIF) neurons (similar to Chizhov and Graham, 2007; Touboul et al., 2011) with approximations for adaptive currents taken from Buchin and Chizhov (2010b):

$$
\begin{gathered}
C_{E} \frac{U_{E}}{d t}=I_{E}-l_{N a}^{L E}-l_{K}^{L E}-l_{C l}^{L E}-g_{A H P} \mathrm{a}\left(U_{E}-V_{A H P}\right)-g_{E E} \mathrm{e} \\
\left(U_{E}-V_{A M P A}\right)-g_{I I} i\left(U_{E}-V_{G A B A}\right) \\
C_{l} \frac{U_{l}}{d t}=-I_{N a}^{L}-l_{K}^{L I}-I_{C l}^{L I}-g_{E I} e\left(U_{l}-V_{A M P A}\right)-g_{I I} i\left(U_{l}-\right. \\
\left.V_{G A B A}\right)
\end{gathered}
$$

where

$$
\begin{aligned}
& I_{N a}^{L E / I}=g_{N a}^{L E / I}\left(U_{E / l}-V_{N a}\right) \\
& I_{K}^{L E / I}=g_{K}^{L E / I}\left(U_{E / l}-V_{K}\right) \\
& I_{C I}^{L E / I}=g_{C I}^{L E / I}\left(U_{E / I}-V_{C I}\right)
\end{aligned}
$$

The firing rate of each population is computed based on the interspike interval distribution of the neural population (Gerstner and Kistler, 2002): 
Table 1. Population model parameters

\begin{tabular}{|c|c|c|}
\hline Parameter & $\begin{array}{l}\text { Excitatory population } \\
\text { Value }\end{array}$ & Interpretation \\
\hline$\overline{C_{E}}$ & $1 \mathrm{mF} / \mathrm{cm}^{2}$ & Membrane capacitance (Buchin and Chizhov, 2010b) \\
\hline$g_{N a}^{L E}$ & $0.02 \mathrm{mS} / \mathrm{cm}^{2}$ & Sodium leak conductance (Krishnan and Bazhenov, 2011) \\
\hline$g_{K}^{L E}$ & $0.044 \mathrm{mS} / \mathrm{cm}^{2}$ & Potassium leak conductance (Krishnan and Bazhenov, 2011) \\
\hline$g_{C l}^{L E}$ & $0.01 \mathrm{mS} / \mathrm{cm}^{2}$ & Chloride leak conductance (Krishnan and Bazhenov, 2011) \\
\hline$g_{A H P}$ & $1.6 \mathrm{mS} / \mathrm{cm}^{2}$ & AHP-current conductance (Buchin and Chizhov, 2010b) \\
\hline$g_{E E}$ & $1.5 \mathrm{mS} / \mathrm{cm}^{2}$ & Excitatory-to-excitatory conductance \\
\hline$g_{E I}$ & $1 \mathrm{mS} / \mathrm{cm}^{2}$ & Excitatory-to-inhibitory conductance \\
\hline$g_{I E}$ & $2 ; 0.5 ; 1 \mathrm{mS} / \mathrm{cm}^{2}$ & Inhibitory-to-excitatory conductance \\
\hline$g_{\|}$ & $0.2 \mathrm{mS} / \mathrm{cm}^{2}$ & Inhibitory-to-inhibitory conductance \\
\hline$U_{\text {reset }}^{E}$ & $-65 \mathrm{mV}$ & Reset membrane potential (Chizhov and Graham, 2007; Buchin and Chizhov, 2010b) \\
\hline$V_{\text {thr }}^{E}$ & $-55 \mathrm{mV}$ & Threshold membrane potential (Chizhov and Graham, 2007; Buchin and Chizhov, 2010b) \\
\hline$a_{E}$ & $2.84 \times 10^{4}$ & Sigmoid fit parameter \\
\hline$b_{E}$ & $0.19 \mathrm{mV}^{-1}$ & Sigmoid fit parameter \\
\hline$c_{E}$ & $1.23 \times 10^{4}$ & Sigmoid fit parameter \\
\hline$d_{E}$ & $-10 \mathrm{mV}$ & Sigmoid fit parameter (threshold) \\
\hline$\sigma_{E}$ & $3 \mu \mathrm{A} / \mathrm{cm}^{2}$ & Input current variance \\
\hline$\tau_{E}$ & $5.4 \mathrm{~ms}$ & AMPA current correlation time (Buchin et al., 2016a,b) \\
\hline$\sigma_{V E}$ & $4 \mathrm{mV}$ & Membrane potential dispersion \\
\hline$V_{\mathrm{Na}}^{\mathrm{E}}$ & $50 \mathrm{mV}$ & Sodium reversal potential (Krishnan and Bazhenov, 2011) \\
\hline$V_{K}^{E}$ & $-75 \mathrm{mV}$ & Potassium reversal potential (Krishnan and Bazhenov, 2011) \\
\hline$V_{C l}^{E}$ & $-93 \mathrm{mV}$ & Chloride reversal potential (Krishnan and Bazhenov, 2011) \\
\hline$V_{G A B A}$ & $-75 \mathrm{mV}$ & GABA reversal potential (Huberfeld et al., 2007) \\
\hline$V_{A M P A}$ & $0 \mathrm{mV}$ & AMPA reversal potential (Brunel and Wang, 2001) \\
\hline$V_{A H P}$ & $-70 \mathrm{mV}$ & AHP reversal potential (Brunel and Wang, 2001) \\
\hline$\tau_{A H P 1}$ & $1 \mathrm{~ms}$ & AHP rise time (Brunel and Wang, 2001) \\
\hline$\tau_{\text {AHP2 }}$ & $320 \mathrm{~ms}$ & AHP decay time (Brunel and Wang, 2001) \\
\hline$\tau_{A M P A 1}$ & $1 \mathrm{~ms}$ & AMPA rise time (Chizhov, 2002) \\
\hline \multirow[t]{2}{*}{$\tau_{A M P A 2}$} & $5.4 \mathrm{~ms}$ & AMPA decay time (Chizhov, 2002) \\
\hline & Inhibitory population & \\
\hline Parameter & Value & Interpretation \\
\hline$C_{1}$ & $1 \mathrm{mS} / \mathrm{cm}^{2}$ & Membrane capacitance (Buchin and Chizhov, 2010b) \\
\hline$g_{\mathrm{Na}}^{L !}$ & $0.02 \mathrm{mS} / \mathrm{cm}^{2}$ & Sodium leak conductance (Krishnan and Bazhenov, 2011) \\
\hline$g_{K}^{L \prime}$ & $0.04 \mathrm{mS} / \mathrm{cm}^{2}$ & Potassium leak conductance (Krishnan and Bazhenov, 2011) \\
\hline$g_{C l}^{L l}$ & $0.03 \mathrm{mS} / \mathrm{cm}^{2}$ & Chloride leak conductance (Krishnan and Bazhenov, 2011) \\
\hline$g_{I E}$ & $2 \mathrm{mS} / \mathrm{cm}^{2}$ & Inhibitory-excitatory synaptic conductance \\
\hline$g_{\|}$ & $0.2 \mathrm{mS} / \mathrm{cm}^{2}$ & Excitatory-inhibitory synaptic conductance \\
\hline$U_{\text {reset }}^{\prime}$ & $-65 \mathrm{mV}$ & Reset membrane potential \\
\hline$V_{\text {thr }}^{\prime}$ & $-55 \mathrm{mV}$ & Threshold membrane potential \\
\hline$a_{1}$ & $2.84 \times 10^{4}$ & Sigmoid fit parameter \\
\hline$b_{1}$ & $0.19 \mathrm{mV}^{-1}$ & Sigmoid fit parameter \\
\hline$c_{l}$ & $1.23 \times 10^{4}$ & Sigmoid fit parameter \\
\hline$d_{1}$ & $-10 \mathrm{mV}$ & Sigmoid fit parameter (threshold) \\
\hline$\sigma_{V I}$ & $4 \mathrm{mV}$ & Membrane potential dispersion \\
\hline$V_{\mathrm{Na}}^{\prime}$ & $50 \mathrm{mV}$ & Sodium reversal potential (Krishnan and Bazhenov, 2011) \\
\hline$V_{K}^{\prime}$ & $-75 \mathrm{mV}$ & Potassium reversal potential (Krishnan and Bazhenov, 2011) \\
\hline$V_{C l}^{\prime}$ & $-82 \mathrm{mV}$ & Chloride reversal potential (Krishnan and Bazhenov, 2011) \\
\hline$\tau_{G A B A 1}$ & $8.3 \mathrm{~ms}$ & GABA-A decay time (Chizhov et al., 2002) \\
\hline$\tau_{G A B A 2}$ & $0.2 \mathrm{~ms}$ & GABA-A rise time (Chizhov, 2002) \\
\hline
\end{tabular}

$$
\nu_{E / /}(t)=A\left(U_{E / l}\right)
$$

where

$$
A(U)=\left[\tau_{m}^{E / I} \int_{\left(U_{\text {reset }}^{E / I}-U_{E / I}\right) / \sigma_{V E / l}}^{\left(V_{\text {threshold }}^{E / I}-U_{E / I}\right) / \sigma_{V E / l}} e^{u^{2}}(1+\operatorname{erf}(u)) d u\right]^{-1}
$$

and

\begin{tabular}{|c|c|}
\hline Variable & Interpretation \\
\hline$U_{E}, \mathrm{mV}$ & $\begin{array}{l}\text { Average membrane potential of the excitatory } \\
\text { population }\end{array}$ \\
\hline$U_{l}, \mathrm{mV}$ & $\begin{array}{l}\text { Average membrane potential of the inhibitory } \\
\text { population }\end{array}$ \\
\hline e & Excitatory population synaptic gating variable \\
\hline$i$ & Inhibitory population synaptic gating variable \\
\hline a & Excitatory population adaptation gating variable \\
\hline$I_{E}(t), \mu \mathrm{A} / \mathrm{cm}^{2}$ & Random excitatory input \\
\hline$\nu_{E}(t), \mathrm{Hz}$ & Firing rate of the excitatory population \\
\hline$\nu_{l}(t), \mathrm{Hz}$ & Firing rate of the inhibitory population \\
\hline
\end{tabular}

Table 2. Population model variables 


$$
\tau_{m}^{E / l}=\frac{C_{E / I}}{g_{N a}^{L E / I}+g_{K}^{L E / I}+g_{C l}^{L E / I}}
$$

In all simulations $\nu_{E / /}(t)$ has been approximated by the following sigmoid function:

$$
\nu_{E / l}(t)=\frac{1}{\tau_{m}^{E / l}} \times \frac{a_{E / /}}{c_{E / l}+\exp \left(-b_{E / /}\left(U_{E / l}+d_{E / l}\right)\right)}
$$

The population firing rate determines the adaptive (a), excitatory (e), and inhibitory (i) gating variables. Their dynamics are computed using the second-order approximation (Wendling et al., 2002; Chizhov, 2014):

$$
\begin{gathered}
\tau_{A H P 1} \tau_{A H P 2} \frac{d^{2} a}{d t^{2}}+\left(\tau_{A H P 1}+\tau_{A H P 2}\right) \frac{d a}{d t}+a=(1-a) \tau \nu_{E} \\
\tau_{A M P A 1} \tau_{A M P A 2} \frac{d^{2} e}{d t^{2}}+\left(\tau_{A M P A 1}+\tau_{A M P A 2}\right) \frac{d e}{d t}+e=(1-e) \tau \nu_{E} \\
\tau_{G A B A 1} \tau_{G A B A 2} \frac{d^{2} i}{d t^{2}}+\left(\tau_{G A B A 1}+\tau_{G A B A 2}\right) \frac{d i}{d t}+i=(1-i) \tau \nu_{l}
\end{gathered}
$$

To mimic the afferent excitatory input, the excitatory population also received stochastic excitatory input modeled as an Ornstein-Uhlenbeck process (Buchin and Chizhov, 2010a):

$$
\tau_{E} \frac{d l_{E}}{d t}=-I_{E}+\sigma_{E} \eta(t)
$$

To mimic elevated extracellular potassium from epileptogenic slice experiments, in the population model, we increased potassium reversal potential in both populations $V_{K}^{E \prime l}$ from -90 to $-75 \mathrm{mV}$, i.e., from $K_{o}=4 \mathrm{mM}$ to $K_{0}=8 \mathrm{mM}$. This value of $V_{K}^{E / l}$ was computed based on Nernst equation, $V_{K}=R T / F \ln \left(K_{\circ} / K_{i}\right)$, where $R T / F=26.64 \mathrm{mV}$ and $K_{i}=138 \mathrm{mM}$ (Krishnan and Bazhenov, 2011).

All model parameter values and variable names are present in Table 1, 2. The initial parameter set was chosen manually to reproduce the pre-ictal like oscillations due to balance between $g_{E E}$ and $g_{A H P}$, seizure and resting state were fit such that $g_{E I}$ parameter variations would make a transition between seizure and resting state.

\section{LFP model}

The LFP was calculated based on the activity of the excitatory population. We assumed that pyramidal cells activity dominates the extracellular field (Buzsáki et al., 2012). The dominant theory is that the LFP component is dominated by the single neuron dipole contribution (Buzsáki et al., 2012). Since the neural mass model averages over single neurons, the dipole moment cannot be directly modeled. Thus, to approximate the LFP being recorded near somas of the excitatory populations, we used the assumption that the average membrane potential of the excitatory population is proportional to the LFP, i.e., LFP $\propto U_{E}$ (Ursino and la Cara, 2006; Demont-Guignard et al., 2009; Wendling et al., 2012; Ratnadurai-Giridharan et al., 2014).

\section{Results}

\section{Construction of the population model}

We developed a model of interacting excitatory and inhibitory population inspired by Wilson-Cowan approach (Wilson and Cowan, 1972), which consists of excitatory and inhibitory populations coupled by synaptic connections (Fig. 1A). The firing rate in each population depends on the average membrane potential $U_{E / /}$, which is governed by the subthreshold dynamics of LIF neuron population similar to (Gerstner and Kistler, 2002; Chizhov, 2014; as explained in Materials and Methods). Firing rates of the excitatory and inhibitory populations are determined using the values of $U_{E / l}$ put through function $A$ $\left(U_{E I I}\right)$ (Johannesma, 1968; Gerstner and Kistler, 2002). To make the model numerically stable and amenable to bifurcation analysis we used a sigmoid function to estimate the population firing rate provided by the $A\left(U_{E / I}\right)$ approximation. To justify the choice of sigmoid parameters, we used least-squares to match it with the analytical solution (Johannesma, 1968; Fig. 1C,D). The sigmoid approximation allows one to efficiently take into account zero and linear parts of the potential-to-rate transfer functions $v_{E / /}$ $(t)$, and provides saturation due to the single neuron refractory period (Renart et al., 2004). The sigmoid functions of excitatory and inhibitory populations are shown in Figure $1 C, D$. The difference between the excitatory and inhibitory populations was taken into account by adjusting passive conductances for sodium, potassium, and chloride leak currents estimated in Krishnan and Bazhenov, (2011) based on dynamic ion concentration model.

The subthreshold $U_{E / /}$ dynamics determine the synaptic $g_{E E} e\left(v_{E}\right), g_{E} e\left(v_{E I I}\right), g_{I I} i\left(v_{l}\right), g_{I E} i\left(v_{l}\right)$, and intrinsic $g_{A H P} e\left(v_{E / I}\right)$ conductances (Fig. 1A), computed according to the population firing-rates $v_{E / / .}$. Similar to spiking neural network models (Bazhenov et al., 2004; Ratnadurai-Giridharan et al., 2014), adaption in our population model reduces neural firing in the excitatory population after periods of activity. Excitatory population receives external random synaptic input to model excitation from the rest of the brain similar to (Jansen and Rit, 1995; Touboul et al., 2011). To mimic the experimental epileptogenic conditions of human subiculum slice experiments, the potassium reversal potential was elevated from -95 to -75 $\mathrm{mV}$ both in the excitatory and inhibitory populations to provide excitatory drive to reproduce the experimental conditions. Elevation of extracellular potassium also leads the increase of intracellular chloride reducing the efficiency of inhibition due to elevated $\mathrm{GABA}_{A}$ reversal potential (Huberfeld et al., 2007; Buchin et al., 2016b). To generate the model output comparable with experimental data, we computed the LFP generated by the excitatory population (Buzsáki et al., 2012). This approximation assumes that all pyramidal cells in the excitatory population contribute equally to the recorded LFP signal (Fig. 1B). Thus, the total LFP near somas depends on the average value of the membrane poten- 
A

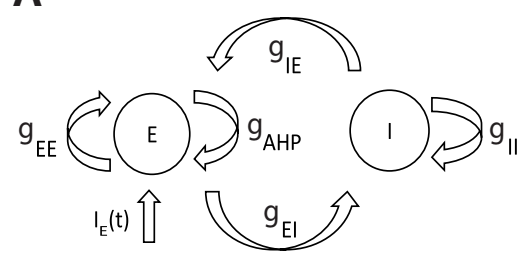

C

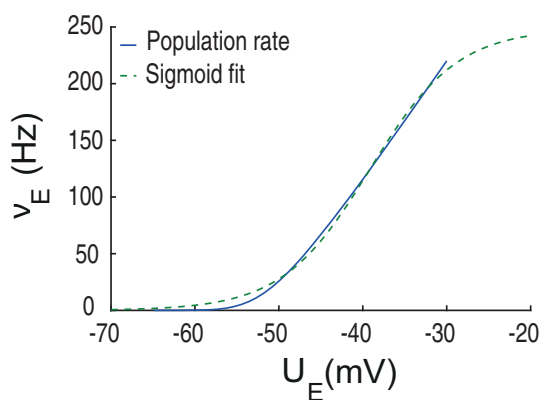

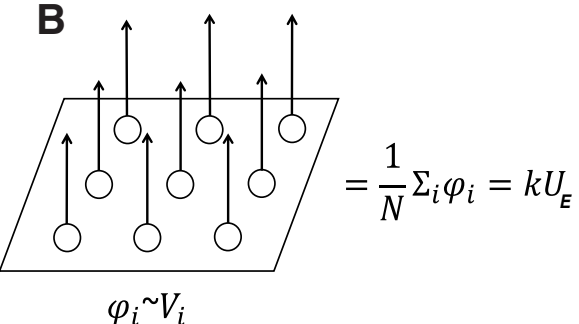

D I population

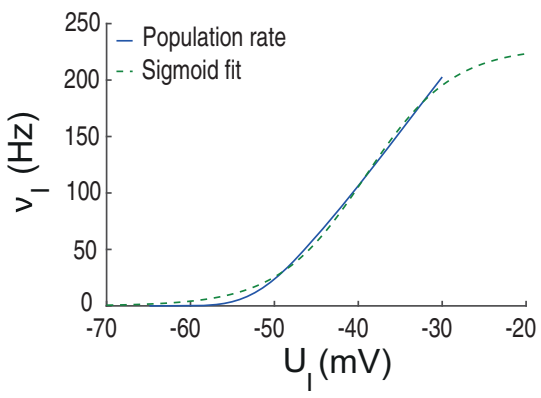

Figure 1. Structure of the population model. $\boldsymbol{A}$, Scheme of interacting neural populations. E, I: excitatory and inhibitory populations; $g_{E E}, g_{E l}$ : excitatory to excitatory and excitatory to inhibitory maximal conductances; $g_{\| l}, g_{I E}$ : inhibitory-to-inhibitory and inhibitory-toexcitatory maximal conductance; $g_{A H P}$ : adaptation conductance in the excitatory population; $I_{E}(t)$ : synaptic noise input to the excitatory population; AHP, afterhyperpolarization current (Buchin and Chizhov, 2010b). B, LFP model: $\varphi_{i}$ : contribution of a single excitatory cell; $N$ : the number of neurons; $U_{E}$ : the average membrane potential in the excitatory population. $\boldsymbol{C}, \boldsymbol{D}$, Sigmoid approximation of potential-to-rate function (Johannesma, 1968) of the excitatory $(\boldsymbol{C})$ and inhibitory population $(\boldsymbol{D})$.

tial in the excitatory population with a certain dimensionality constant, i.e., $L F P \propto k U_{E / /}$.

\section{Reproduction of epileptic oscillations}

When the excitatory and inhibitory synaptic currents were dynamically balanced, the activity stayed in the low-firing regime, as indicated by LFP power spectrum (Fig. 2). The recorded pyramidal cell during this period demonstrated sparse firing activity, partially time-locked with the discharges on the LFP. We call this activity in the model the balanced or resting state (Fig. $2 A$ ). In this regime, the model does not generate epileptic oscillations. To evaluate the model performance in this resting state, we compared the synthetic LFP with the experimental LFP recorded between seizures (Fig. 2A). Similar to the experimental data, we found that in the resting state, the model generates broadband oscillations, with the highest power in the $1-$ to $15-\mathrm{Hz}$ frequency band. In this regime, the average membrane potential of the excitatory population $U_{E / /}$ stays in the range from -60 to -50 $\mathrm{mV}$.

We found that the model was not capable of generating interictal discharges using this parameter set. It has been recently suggested that interneurons play the key role in generating interictal activity (Cohen et al., 2002; Huberfeld et al., 2011). In the presence of $\mathrm{GABA}_{\mathrm{A}}$ blockade these events were completely blocked, indicating that they depend on combination of GABAergic and glutamatergic signaling. In the recent population model (Chizhov et al., 2017), it was proposed that interictal discharges could be initiated by the inhibitory population, thus explaining in- terneuron firing before pyramidal cell firing (Huberfeld et al., 2011). In our model we have not explored this scenario, i.e., when the inhibitory population is also receiving the background synaptic input. These mechanisms would likely play an important role for seizure initiation; however, incorporating all mechanisms at once would make the model impossible to study analytically. Therefore, we have not considered interictal discharges before seizure, while aiming to specifically describe other types of oscillations.

To reproduce the seizure state in the model, we reduced the synaptic inhibition of the excitatory population by decreasing the synaptic conductance parameter $g_{I E}$ (Fig. 2B, black arrow). All other parameters of the model remained the same. In this case the model moved into an oscillatory regime in which the power spectrum of the oscillations changed dramatically to include strong oscillations in the 1- to $4-\mathrm{Hz}$ frequency band, which is typical for ictal discharges (Huberfeld et al., 2011).

We compared the model power spectrum with the measured LFP recorded during the initial phase of the ictal discharge with the hypersynchronous activity onset. During this activity regime the recorded pyramidal cells generated strong bursts of spikes temporally locked to the LFP (Fig. 2B). The population model displayed discharges with the same frequency band as in the LFP, indicating large amount of synchrony in the excitatory population (Buzsáki et al., 2012). Note that we considered only the initial phase of the seizure (the whole ictal event is shown in Fig. 3E). 

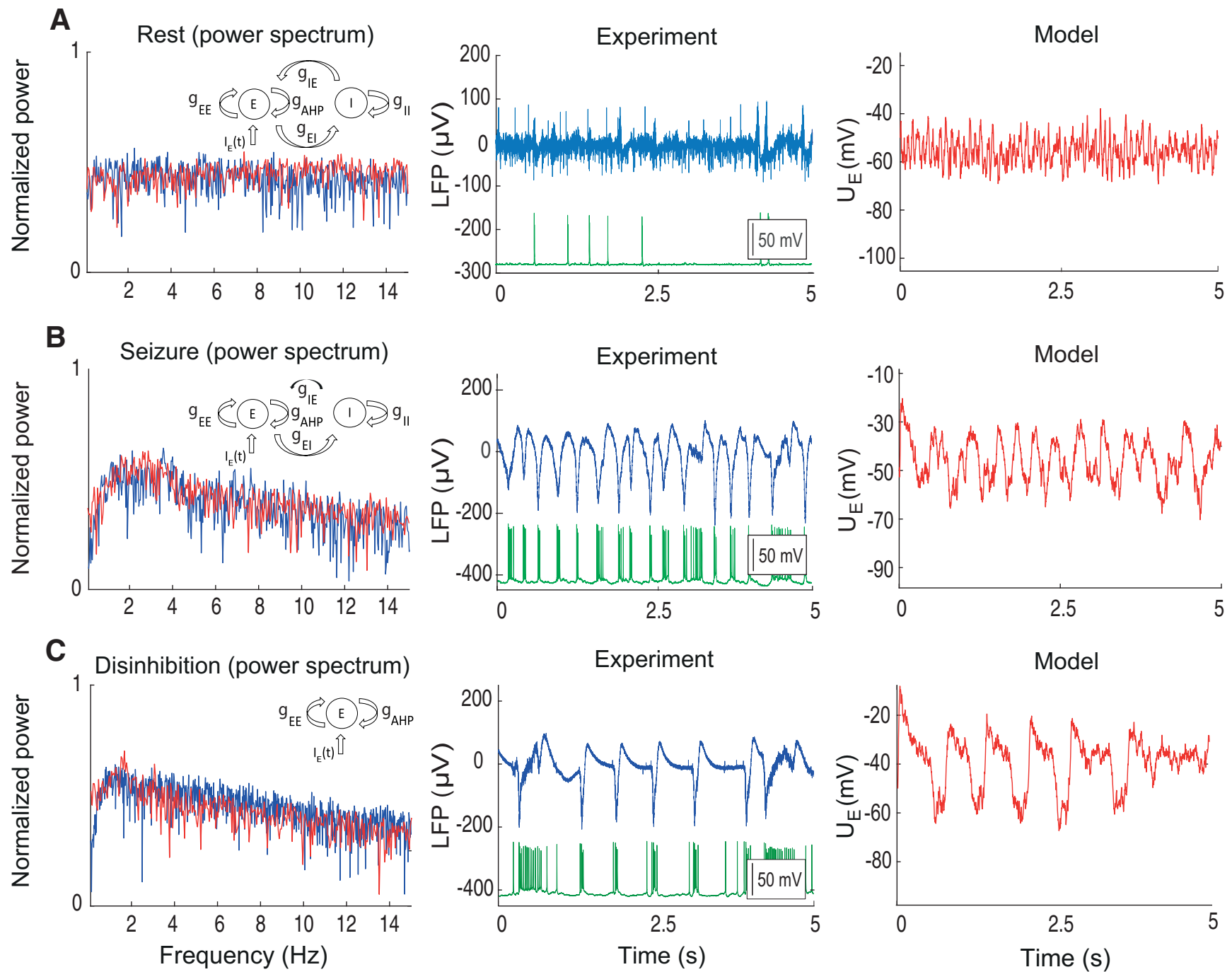

Figure 2. Neural mass model in various excitatory regimes. $\boldsymbol{A}$, Activity of a neural population in the resting state. $\boldsymbol{B}$, Seizure state. $\boldsymbol{C}$, Disinhibited state. LFP is present together with intracellular recording from the pyramidal cell. Each plot contains the model scheme, power spectrum, and time traces provided by the excitatory population $U_{E}$ as well as experimental LFP. Red traces correspond to the model, blue traces to the experiment, and green traces to the intracellular recordings from the pyramidal cells. Model parameters for $(\boldsymbol{A}): g_{E E}=1.5 \mathrm{mS} / \mathrm{cm}^{2} ; g_{E I}=1 \mathrm{mS} / \mathrm{cm}^{2} ; g_{I E}=2 \mathrm{mS} / \mathrm{cm}^{2} ; g_{I I}=0.2 \mathrm{mS} / \mathrm{cm}^{2} ; g_{A H P}=1.6 \mathrm{mS} / \mathrm{cm}^{2} ;(\boldsymbol{B}): g_{E E}=1.5 \mathrm{mS} / \mathrm{cm}^{2} ; g_{E I}=1 \mathrm{mS} / \mathrm{cm}^{2}$; $g_{I E}=0.5 \mathrm{mS} / \mathrm{cm}^{2} ; g_{I I}=0.2 \mathrm{mS} / \mathrm{cm}^{2} ; g_{A H P}=1.6 \mathrm{mS} / \mathrm{cm}^{2} ;(\boldsymbol{C}): g_{E E}=1.5 \mathrm{mS} / \mathrm{cm}^{2} ; g_{E I}=1 \mathrm{mS} / \mathrm{cm}^{2} ; g_{I E}=0 \mathrm{mS} / \mathrm{cm}^{2} ; g_{I I}=0.2 \mathrm{mS} / \mathrm{cm}^{2}$; $g_{\text {AHP }}=1.6 \mathrm{mS} / \mathrm{cm}^{2}$.

To further test the validity of our model, we explored its dynamics with inhibitory activity completely blocked (Fig. $2 C)$. In these simulations the initial conditions were set to the resting state and parameter values of the model were set to the seizure state, but with the conductance $g_{I E}$ (from the inhibitory to the excitatory population) set to zero to mimic the experimental conditions. In this case the GABAergic effects of the inhibitory population in the slice has been fully blocked by bicuculine after seizures have been previously established (Huberfeld et al., 2011). In response to this change, the activity in the slice became highly synchronized and reduced to regular pre-ictal discharges. During these oscillations the pyramidal cells generated large bursts of activity, temporally coupled with the LFP (Fig. 2C). In the model, similarly to the experimental preparation, the blockade of the GABAergic signaling mimicked by the abolition of the inhibitory population led to the development of a slow oscillatory rhythm with a peak frequency around $1 \mathrm{~Hz}$. These events have been previously reported as pre-ictal discharges (Huberfeld et al., 2011). This rhythm has much slower frequency than seizures, and is usually within the $1-$ to $4-\mathrm{Hz}$ frequency range (Huberfeld et al., 2011; Buchin et al., 2016b). In addition, these events recur regularly for long periods with very limited modulation.

We call this regime of activity pre-ictal discharges because similar activity takes place before transition toward an ictal state (Huberfeld et al., 2011). In this regime, the dynamics of the excitatory population are determined only by the balance between self-excitation, $g_{E E} e\left(v_{E / I}\right)$, afterhyperpolarization current (AHP; Chizhov and Graham, 2008; Buchin and Chizhov, 2010b), $g_{A H P} e\left(v_{E I I}\right)$, and the afferent 
A
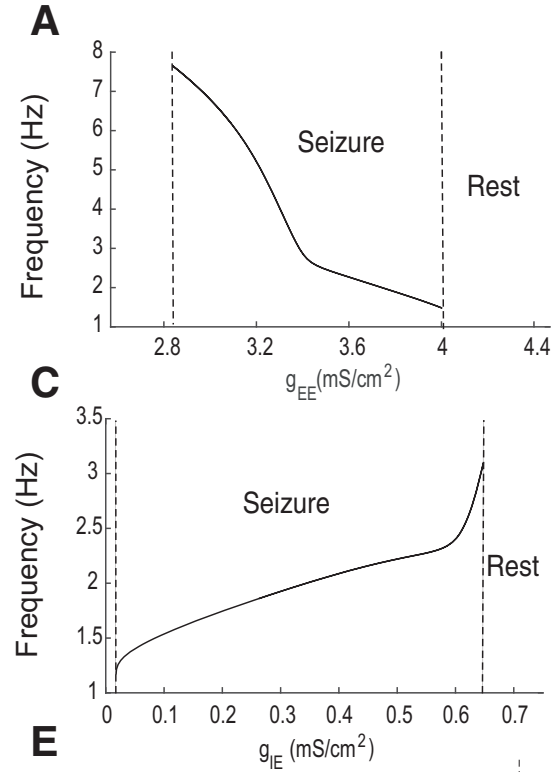

हो
总
产
$>$
B
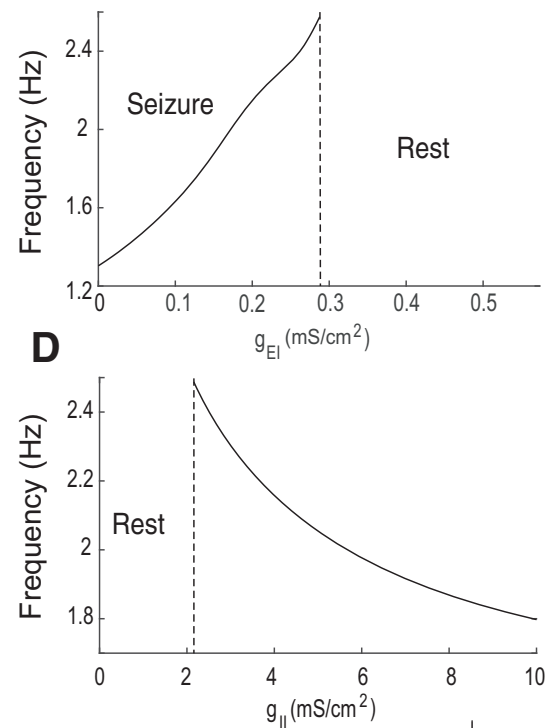

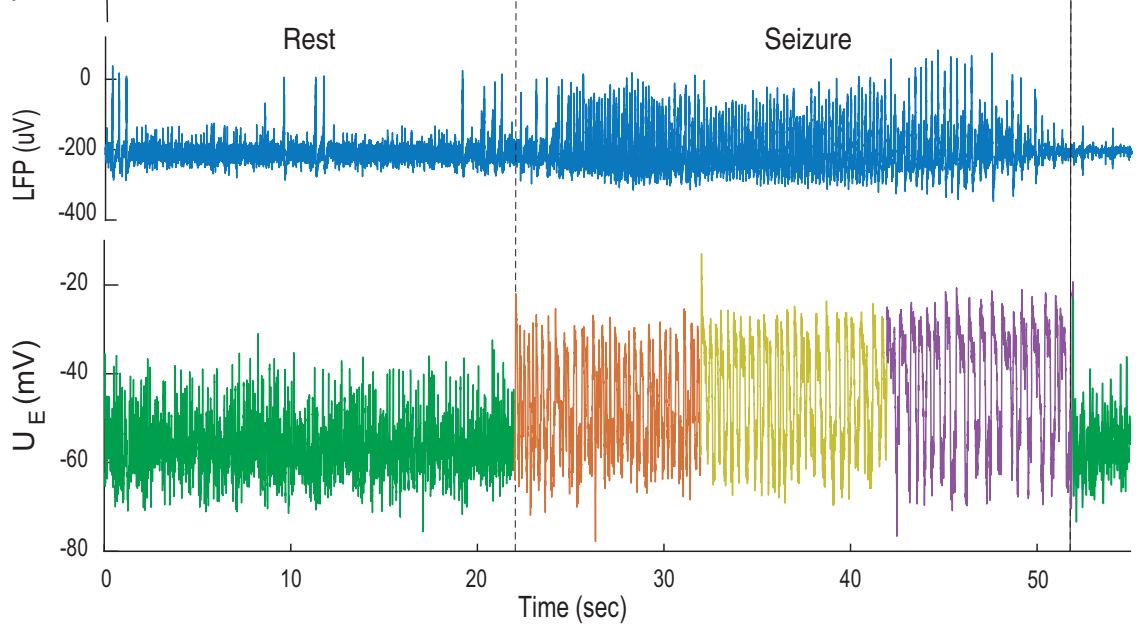

Figure 3. Oscillatory frequencies of the population model. $\boldsymbol{A}-\boldsymbol{D}$, Oscillatory frequencies of the population model in the absence of the synaptic noise $\left(I_{E}(t)=0\right)$ as a function of the synaptic conductance, $g_{E E}, g_{E l}, g_{I E}, g_{I \mid}$. E. Simultaneous intracellular recording from single pyramidal cell, LFP, and population model during transition from the resting state toward seizure. States marked by dotted lines. The green trace corresponds to the model's resting state (Fig. $2 A, g_{I E}=2 \mathrm{mS} / \mathrm{cm}^{2}$ ), red corresponds to early seizure (Fig. $2 B$, $g_{I E}=$ $\left.0.5 \mathrm{mS} / \mathrm{cm}^{2}\right)$, yellow corresponds to late seizure $\left(g_{I E}=0.25 \mathrm{mS} / \mathrm{cm}^{2}\right)$, and purple corresponds to the disinhibition state (Fig. $2 C$, $\left.g_{\text {IE }}=0 \mathrm{mS} / \mathrm{cm}^{2}\right)$.

synaptic current $I_{E}(t)$. Hence, these pre-ictal oscillations in the model are driven by the synaptic noise and adaptation. The excitatory input to the excitatory population $I_{E}$ $(t)$ drives the upswings of $U_{E}$ due to recurrent excitatory synapses, with activity then being terminated by AHP currents. These transitions take place randomly due to stochastic nature of the synaptic input.

For quantitative comparisons between the model and experiment we used the linear fit to the power spectrum over frequencies and peak estimation (Table 3). We found that there is substantial intersection between linear fits applied to the power spectrums in resting, seizure, and pre-ictal states (Fig. 2). We found that there is a substantial overlap between these frequencies, providing validation for the model. Note that we compared the overall spectral characteristics between the model and experiment by variation of only one parameter, $g_{I E}$ to reproduce transitions between the pre-ictal, resting and seizure states. If more parameters are varied at the same time, it would be possible to get a better match between the model and experiment.

Overall oscillations in our population model are controlled by the balance between synaptic currents, adaptation and external synaptic input. When synaptic and 
Table 3. Power spectrum analysis

\begin{tabular}{lllll}
\hline & Model, peak & Experiment, peak & Model, spectrum & $\begin{array}{l}\text { Experiment, spectrum } \\
\text { amplitude, } \mathrm{Hz}\end{array}$ \\
Rest & - & - & linear fit, $1 / \mathrm{Hz}$ & $-0.005-0.002$ \\
Seizure & $3.01-3.52$ & $2.95-3.75$ & $-0.005-0.002$ & $-0.003-0.002$ \\
Pre-ictal state & $1.33-1.43$ & $1.21-1.79$ & $-0.005-0.002$ & $-0.01--0.008$ \\
\hline
\end{tabular}

intrinsic conductances are balanced, the population demonstrates resting state activity, characterized by a flat power spectrum. When there is an imbalance between excitation and inhibition, populations start developing oscillatory rhythms associated with ictal discharges with a frequency of 3-4 Hz. However, complete loss of inhibition leads to the development of another population rhythm, pre-ictal discharges with $1-\mathrm{Hz}$ frequency, controlled by adaptation and recurrent excitation. Thus, the dynamic state of a neural population depends on the interplay between the intrinsic and synaptic excitability within populations as well as external synaptic input.

\section{Analysis of the population model}

To delineate the mechanisms giving rise to the different oscillatory modes in the model, we used continuation techniques and bifurcation analysis. Since it is impossible to use the standard techniques to identify bifurcations in the presence of noise, we analyzed the model in the absence of an external input $I_{E}(t)$. This allowed us to compute the model behavior in the stationary regime and characterize bifurcations happening during transitions between different oscillatory regimes. The initial parameters were chosen to correspond to the resting state. The parameter variations were calculated around this point in the parameter space for $g_{E E}, g_{E I}, g_{I E}$, and $g_{\mid l}$ bifurcation diagrams, with other parameters held fixed. Analysis of $g_{A H P}$ and $V_{G A B A}$ variations was implemented for another parameter set, where $g_{I E}=0.5 \mu \mathrm{S} / \mathrm{cm}^{2}$ and $g_{I E}=1$ $\mu \mathrm{S} / \mathrm{cm}^{2}$; other parameters remained the same.

The frequency of seizure oscillations depends on the strength of the synaptic currents in the population model. There is a nonlinear relationship between seizure major frequency and the recurrent excitatory conductance $g_{E E}$ (Fig. 3A). When the $g_{E E}$ is increased up to $2.8 \mathrm{mS} / \mathrm{cm}^{2}$, the model responds with an oscillatory frequency near $7.5 \mathrm{~Hz}$. When self-excitation is further increased up to $4 \mathrm{mS} / \mathrm{cm}^{2}$, seizure-related oscillations disappear since the system moves to the high activity state due to sigmoidal saturation of the transfer function (Fig. 1C,D). The amount of stimulation of the inhibitory population also influences the oscillatory frequency. When $g_{E I}$ is in the range of 0 to 0.29 $\mathrm{mS} / \mathrm{cm}^{2}$ (Fig. 3B), the population model generates seizure activity with frequencies of $1.2-2.5 \mathrm{~Hz}$. Note that seizure oscillations are possible even when $g_{E I}=0 \mathrm{mS} / \mathrm{cm}^{2}$.

Inhibitory synaptic connections also affect the oscillatory frequency of seizure activity. When $g_{I E}$ is as low as $\sim 0.6 \mathrm{mS} / \mathrm{cm}^{2}$ (Fig. $3 C$ ), the seizure activity starts around $3 \mathrm{~Hz}$; it decreases to $\sim 1 \mathrm{~Hz}$ when $g_{E I}$ is close to zero (when $g_{I E}=0 \mathrm{mS} / \mathrm{cm}^{2}$, there is no seizure activity in the model). The amount of recurrent inhibition also determines the seizure oscillatory frequency (Fig. $3 D$ ). Seizure activity can be initiated by sufficient self-inhibition, i.e., when $g_{\|}$is near $2 \mathrm{mS} / \mathrm{cm}^{2}$, seizures of $2.5 \mathrm{~Hz}$ are observed. When $g_{\|}$increases, the seizure frequency decreases; for example, at $10 \mathrm{mS} / \mathrm{cm}^{2}$, seizure activity is $\sim 1.8 \mathrm{~Hz}$.

In the previous sections, the population model was calibrated to data for short periods of seizure activity, where the frequency was not substantially changing (Fig. $2 B$ ). Yet, one can see that in the experiment, seizure activity is not stationary and its frequency changes over time. The time course of a typical seizure is shown in Figure $3 E$. Before the seizure starts there is a resting state, characterized by occasional interictal (Cohen et al., 2002) and pre-ictal discharges (Huberfeld et al., 2011). When seizure starts at $22 \mathrm{~s}$, it is characterized by fast oscillations of the extracellular field in the range of $5-6 \mathrm{~Hz}$ in the initial phase. During the time course of seizure activity, it gradually decreases to $1-\mathrm{Hz}$ frequency, and from $52 \mathrm{~s}$, it gradually stops.

We aimed to reproduce this aspect of seizure activity using the population model (Fig. 3E). First, the model was initialized in the resting state (Fig. $2 A$ ), green trace. Second, we reduced the amount of inhibitory-to-excitatory coupling (to $g_{I E}=0.5 \mathrm{mS} / \mathrm{cm}^{2}$ ) to reproduce the seizure state, red trace. Third, we gradually reduced the coupling parameter (to $g_{I E}=0.25 \mathrm{mS} / \mathrm{cm}^{2}$ ) to reduce the oscillation frequency, yellow trace. Fourth, to model the slow oscillations in the end of seizure, we set the coupling parameter to zero $\left(g_{I E}=0 \mathrm{mS} / \mathrm{cm}^{2}\right)$, violet trace. Finally, we restored it to the original value to bring the model back to rest $\left(g_{I E}=2 \mathrm{mS} / \mathrm{cm}^{2}\right)$, green trace. This example illustrates how the transition toward seizure in the population model can be achieved by varying only one parameter, the inhibitory-to-excitatory conductance $g_{I E}$.

To study the amplitude of pathologic oscillations, we performed a bifurcation analysis and tracked changes of the average membrane potential in the excitatory population, $U_{E}$ (Fig. 4), the self-excitation conductance $g_{E E}$ (Fig. $4 A)$. We found that increasing $g_{E E}$ leads to the development of ictal oscillations when its value increases beyond $\sim 2.8 \mathrm{mS} / \mathrm{cm}^{2}$. During the gradual increase of $g_{E E}$, the constant steady state loses stability via the supercritical Hopf bifurcation (Izhikevich, 2007), red dot. After passing this point the neural populations start developing seizure oscillations. This activity regime is stable for large $g_{E E}$ variations, implying that seizure dynamics are possible for a large range of recurrent excitation. When $g_{E E}$ becomes higher than a critical value $\left(>4.1 \mathrm{mS} / \mathrm{cm}^{2}\right)$ and the system loses stability via the subcritical Hopf bifurcation, green dot. It corresponds to the high activity state with no oscillations. This happens due to the sigmoid approximation of the population rate (Johannesma, 1968), when $\nu_{E / I}$ reaches the saturation level (Fig. 1C,D). 
A
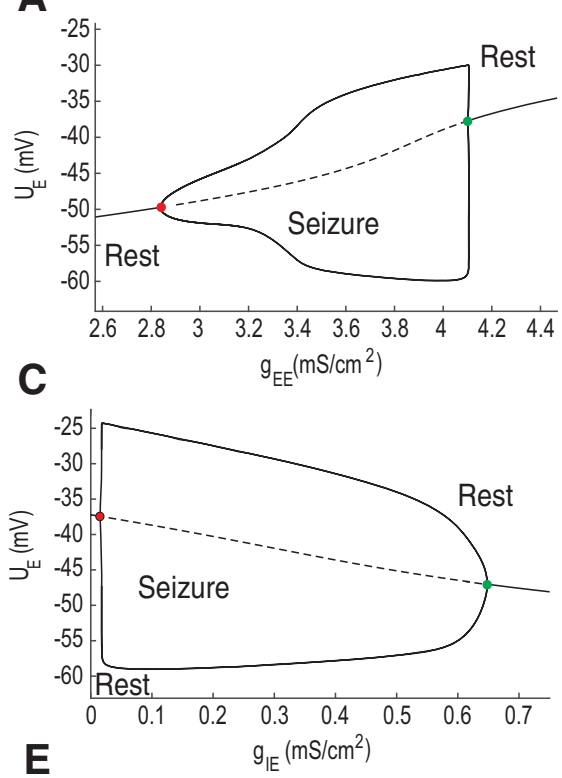

E

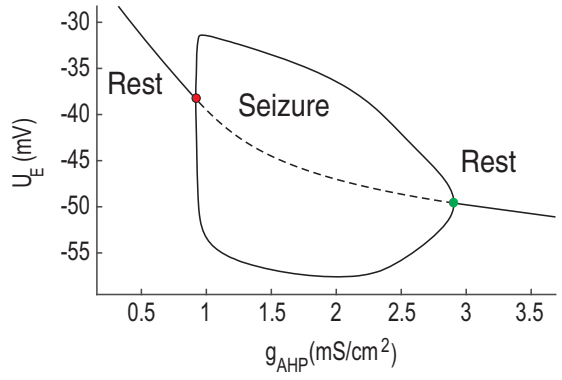

B

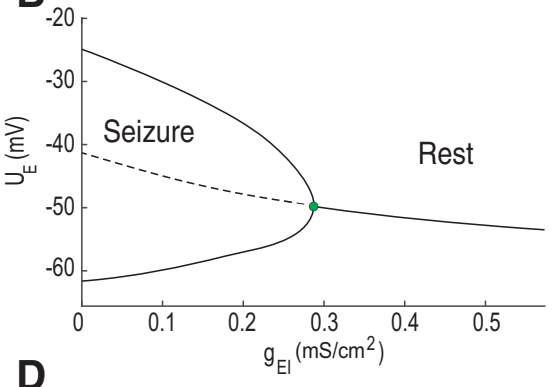

D
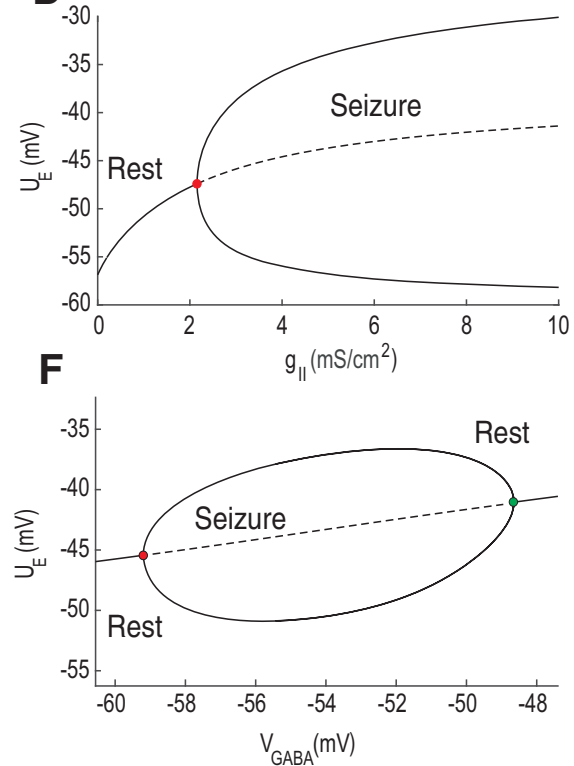

Figure 4. Analysis of the population model. $\boldsymbol{A}-\boldsymbol{D}$, Bifurcation diagrams for the variations of the maximal synaptic conductances, including recurrent excitation $g_{E E}$, excitation from excitatory to inhibitory population $g_{E I}$, inhibition from inhibitory to excitatory population $g_{I E}$, and the recurrent inhibition in the inhibitory population $g_{\|}$, respectively. $\boldsymbol{E}, \boldsymbol{F}$, Bifurcation diagrams for adaptation in the excitatory population $g_{A H P}$ and GABA reversal potential $V_{G A B A}$ from the inhibitory-to-excitatory current, $g_{I E} i\left(U_{E}-V_{G A B A}\right)$. Diagrams $A-D$ were calculated for $g_{I E}=2 \mathrm{mS} / \mathrm{cm}^{2} ; \boldsymbol{E}, g_{I E}=0.5 \mathrm{mS} / \mathrm{cm}^{2}$; and $\boldsymbol{F}, g_{I E} 1 \mathrm{mS} / \mathrm{cm}^{2}$. The value of $U_{E}$ characterizes the average membrane potential in the resting state and maximal/minimal values of $U_{E}$ during the oscillations. Red and green dots correspond to the supercritical and subcritical Andronov-Hopf bifurcations. Solid and dotted lines depict the stable and unstable solutions.

Second, we considered the excitatory to inhibitory conductance $g_{E l}$ (Fig. $4 B$ ). In this case, seizure activity is blocked when $g_{E I}$ is larger than $0.3 \mathrm{mS} / \mathrm{cm}^{2}$. If $g_{E I}$ is smaller than $0.3 \mathrm{mS} / \mathrm{cm}^{2}$, it leads to seizure activity via a subcritical Hopf bifurcation, green dot. Similar to the $g_{E E}$ bifurcation diagram, seizure dynamics are possible for a large range of $g_{E I}$. These results show that a decrease in the excitatory conductance from excitatory to inhibitory populations is sufficient to provoke seizure activity. Note that even if $g_{E l}=0 \mathrm{mS} / \mathrm{cm}^{2}$, the excitatory population still receives the input from the inhibitory one because potassium reversal potential is elevated. These changes in potassium reversal potential drive both excitatory and inhibitory population even if synaptic drive is not present. For example, when $g_{E I}=0 \mathrm{mS} / \mathrm{cm}^{2}$, the increased potassium reversal potential still drives the inhibitory population, providing the inhibitory input to the excitatory population. It happens because it decreases the leak current thus depolarizing the membrane potential of excitatory and inhibitory neurons. Therefore, seizure oscillations are still present because inhibition is still present.
Seizure frequency in this case is near $1.25 \mathrm{~Hz}$ (Fig. 3B) and $U_{E}$ oscillates between -61 and $-25 \mathrm{mV}$.

Third, we considered inhibitory-to-excitatory conductance $g_{I E}$ (Fig. 4C). When $g_{I E}=0$, the model shows resting state activity. This corresponds to the condition when the inhibitory population does not have any influence on the excitatory one. Experimentally this scenario is achieved when inhibitory neurotransmission is completely blocked. Therefore, in the complete absence of inhibition, seizure activity could not be generated. In turn, pre-ictal oscillations are not possible without the contribution of the external synaptic noise $I_{E}(t)$ when $g_{I E}=0 \mathrm{mS} / \mathrm{cm}^{2}$. When there is stochastic synaptic input, it occasionally brings the system to the oscillatory regime associated with seizures (Fig. 2C). Then oscillations are promoted due to recurrent excitation and terminated via AHP adaptation mechanism. Thus, without synaptic stimulatin of the inhibitory population, the model is incapable of seizure generation. In turn, pre-ictal oscillations do not require inhibition, but strongly depend on the recurrent excitatory-toexcitatory connections $g_{E E}$, adaptation $g_{A H P}$, and the external 
synaptic input $I_{E}(t)$. When inhibitory to excitatory conductance $g_{I E}$ becomes strong enough, around $0.65 \mathrm{mS} / \mathrm{cm}^{2}$, seizure oscillations become truncated and the system moves back to the resting state via subcritical Andronov-Hopf bifurcation, green dot.

Fourth, we evaluated the role of recurrent inhibitory conductance $g_{\|}$for seizure dynamics (Fig. 4D). When there is substantial amount of self-inhibition in the inhibitory population, it leads to an increase of excitation in the whole system because of synaptic coupling. If $g_{\text {/I }}$ is above $2.1 \mathrm{mS} / \mathrm{cm}^{2}$, it leads to the development of seizure oscillations via a supercritical Hopf bifurcation, red dot. Seizure activity in this case persists for the large variations in $g_{\|}$variations, from 2.2 to $>10 \mathrm{mS} / \mathrm{cm}^{2}$.

We then analyzed the effect of adaptation in the excitatory population. We found the regime in the parameter space of the model for which $g_{A H P}$ becomes the critical parameter for seizure oscillations. To find this regime we slightly modified the parameter set, where $g_{I E}=0.5 \mathrm{mS} /$ $\mathrm{cm}^{2}$ instead of $2 \mathrm{mS} / \mathrm{cm}^{2}$. In this case, $g_{A H P}$ could substantially affect seizure oscillations. When $g_{A H P}$ is in the range of $1-3 \mathrm{mS} / \mathrm{cm}^{2}$, there is a large region in the parameter space that produces seizure oscillations. If $g_{A H P}$ is larger than $3 \mathrm{mS} / \mathrm{cm}^{2}$, the seizure dynamics becomes truncated due to the inhibitory effect of adaptation via subcritical Andronov-Hopf bifurcation, green dot. Yet when adaptation is not strong enough, $g_{A H P}$ is lower, the model demonstrates seizure oscillations. If $g_{A H P}$ is lower than $1 \mathrm{mS} / \mathrm{cm}^{2}$, seizure oscillations become impossible and the model moves to the high activity state without oscillations via supercritical Andronov-Hopf bifurcation. Additionally, we found that in the complete absence of adaptation, seizure oscillations are still possible in the model (results not shown), but pre-ictal oscillations could not be generated because of the absence of negative feedback provided by adaptation. To be able to reproduce seizure oscillations together with pre-ictal oscillations induced by $\mathrm{GABA}_{\mathrm{A}}$ blockade, adaptation in the excitatory population is required.

We further studied the critical role of $V_{G A B A}$ for seizure generation. It has been recently found that changes in $V_{G A B A}$ are associated with the rhythm generation in the hippocampus (Cohen et al., 2002; Huberfeld et al., 2007). The analysis was performed for slightly modified parameter set, where $g_{I E}=1 \mathrm{mS} / \mathrm{cm}^{2}$, such that $V_{G A B A}$ becomes the bifurcation parameter. The other parameters remained the same. We have changed the initial parameter set to find the region of the parameter space where $V_{G A B A}$ could play the crucial role for oscillations. We found that when $V_{G A B A}$ is higher than $-59 \mathrm{mV}$, it leads to ictal oscillations (Fig. 4F). If $V_{\text {GABA }}$ drops below $-48 \mathrm{mV}$, the oscillations stop. These transitions take place due to supercritical and subscritical Andronov-Hopf bifurcations. Thus, there is substantial range of $V_{G A B A}$ where its increase leads to the development of seizures, which might take place due to chloride accumulation before an ictal discharge (Huberfeld et al., 2007; Lillis et al., 2012).

In summary, using bifurcation analysis, we characterized the parameter regions of the model where seizure oscillations could take place. We found that transitions from seizure to rest and from rest to seizure take place via supercritical and subcritical Andronov-Hopf bifurcations. In all studied cases we found that resting and oscillatory solutions exist for large parameter variations, implying the stability of found solutions (Prinz et al., 2004; Marder and Taylor, 2011). We showed that variations of synaptic $g_{E E}$, $g_{E I}, g_{I E}, g_{I l}$, and intrinsic conductances $g_{A H P}$ could bring the system toward seizure and move it back to the resting state. It implies that combination of recurrent synaptic currents and spike-frequency adaptation in the excitatory population accounts for the transitions between seizure and resting states.

\section{Discussion}

The objective of this study was to investigate the role of intrinsic excitability and inhibition as mechanisms of seizure dynamics. We constructed a novel neural mass model, consisting of interacting excitatory and inhibitory neural populations driven by external synaptic input. By comparing the model with the LFP data from human hippocampal/subicular slices, we found that it could accurately represent resting states, ictal discharges, and pre-ictal oscillations after the blockade of inhibition (Huberfeld et al., 2011). Analysis of the model showed that synaptic and intrinsic conductances play the most crucial role for transitions between resting and seizure activity. By analyzing the parameter space of the model, we found the oscillatory regimes specific for the resting state and seizure dynamics, and found that transitions between these regimes take place via subcritical and supercritical Andronov-Hopf bifurcations.

Starting with the pioneering work of Wilson and Cowan (Wilson and Cowan, 1972), neural mass models have traditionally aimed to reduce the complexity of neural dynamics toward interactions between excitation and inhibition. This approach has been validated in multiple studies for describing the large-scale brain activity patterns (Jirsa et al., 2010). Additionally, it has been shown that intrinsic properties of single neurons such as spikefrequency adaptation (Fröhlich et al., 2008) substantially change spiking patterns and thus neural dynamics (Kager et al., 2000; Bazhenov et al., 2004; Buchin et al., 2016b). So far these types of interactions have not been explicitly taken into account in neural mass models.

In this work, we developed a novel mass model by adding AHP currents (Buchin and Chizhov, 2010a) to the excitatory population. This allowed to efficiently take into account not only seizure and resting state dynamics (Wendling et al., 2012) but also pre-ictal oscillations. In our model seizure activity takes place due to imbalance between self-excitation, adaptation and inhibition. We found that reducing the amount of inhibition to the excitatory population provokes seizure activity. Nonetheless, inhibition plays an important role in orchestrating seizures as well (Fig. 2B). We found that the complete lack of inhibition leads to the development of slow oscillations with significantly different frequency content than seizures (Fig. 2C). Thus, we propose that inhibition, together with single neuron intrinsic properties provided by adaptation, plays an important role controlling the seizure dynamics. 
We have investigated multiple mechanisms responsible for generation of seizure activity. In the proposed model, seizure oscillations could be generated by increased recurrent excitation $g_{E E}$, decreased excitation of the inhibitory population $g_{I E}$, decreased inhibition of the excitatory population $g_{I E}$, increased recurrent inhibition in the inhibitory population $g_{\|}$. Changes in the intrinsic excitability of the excitatory population such as decrease of intrinsic adaptation $g_{A H P}$ and increase of the $\mathrm{GABA}_{\mathrm{A}}$ reversal potential $V_{G A B A}$ could also lead to seizure oscillations. We speculate that various physiological parameters combinations could lead to seizure activity, as found by Jirsa et al. (2014). The combination of multiple factors such as increased chloride concentration in the pyramidal cells and $\mathrm{GABA}_{\mathrm{A}}$ reversal (Huberfeld et al., 2011; Lillis et al., 2012; Buchin et al., 2016a), together with an increase in extracellular potassium concentrations (Bazhenov et al., 2004; Krishnan and Chizhov, 2011) and decreased activity of interneurons (Ziburkus et al., 2006), all contribute to seizure initiation. Combination of these factors and their relative contribution should be evaluated via additional experiments and modeling.

Adaptation on the single neuron level could be achieved by calcium-dependent potassium currents (Jung et al., 2001; Bazhenov et al., 2004). In our model, AHP is the key mechanism for termination of population bursts during seizure oscillations (Fig. 2B) and pre-ictal discharges (Fig. $2 C)$. The alternative potential mechanism of termination of these bursts are $\mathrm{GABA}_{B}$ currents provided by the inhibitory population (de la Prida et al., 2006). We predict that in the complete absence of the inhibitory neurotransmission including $\mathrm{GABA}_{A}$ and $\mathrm{GABA}_{B}$ synapses, the purely excitatory network in the epileptogenic slice of human subiculum would be capable of generating self-sustained preictal oscillations due to negative feedback provided by AHP (Ratnadurai-Giridharan et al., 2014) and other intrinsic adaptation currents. Therefore the downregulation of excitatory neuronal adaptation currents such as AHP and/or functionally similar muscarinic-sensitive potassium currents (Stiefel et al., 2008) could lead to seizure initiation. According to the model the pharmacological strategy aiming to increase the amount of adaptation in the excitatory population would lead to the decreased susceptibility toward seizures.

$\mathrm{GABA}_{B}$ inhibition could also participate for the termination of population bursts. As shown by (de la Prida et al., 2006), the joint blockade of GABA $A_{A}$ receptors by PTX and $\mathrm{GABA}_{\mathrm{B}}$ receptors by CGP led to generation of all-or-none population bursts in CA3 mouse hippocampal slices. In our experiments we did not test for the possibility that $\mathrm{GABA}_{\mathrm{B}}$ could participate for the pre-ictal discharge termination. Additional experiments are needed to divide the contributions of $\mathrm{GABA}_{\mathrm{B}}$ and $\mathrm{AHP}$ for the burst termination.

Note that oscillations in the slice switched from ictal discharges to pre-ictal ones after full $\mathrm{GABA}_{\mathrm{A}}$ blockade. This transition was possible only if seizures were already established in the slice (Huberfeld et al., 2011). It implies that there are excitability and synaptic plasticity changes in the slice associated with seizures before the pre-ictal discharges could be established using complete $\mathrm{GABA}_{\mathrm{A}}$ blockade. When $\mathrm{GABA}_{A}$ blockers were applied before first seizure being generated, the pre-ictal and ictal oscillations were not established (Huberfeld et al., 2011).

Our model has several limitations compared to existing approaches (Molaee-Ardekani et al., 2010; Wendling et al., 2012; Jirsa et al., 2014). First, it is unable to describe the pre-ictal discharges taking place before seizure. The work of Buchin et al. (2016b) proposes a network explanation of pre-ictal discharges that take place before seizure transition (Huberfeld et al., 2011). To describe this activity, it was necessary to take into account the heterogeneity in the excitatory population caused by depolarizing GABA, while in the current model we did not take it into account. Therefore, pre-ictal discharges in our model could be generated only in the absence of inhibitory population. Second, particular features such as high frequency oscillations (Engel et al., 2009) relevant for seizure initiation (Quilichini et al., 2012) are not captured in our model. We speculate that this property could be taken into account by incorporating fast somatic and slow dendritic inhibition (Wendling et al., 2012). Third, our model is also unable to describe the interictal discharges, which have been explained in the other population models (Wendling et al., 2012; Chizhov et al., 2017). It has been found that interictal discharges in human subiculum require initial interneuron activation. Since in our model we impose the background synaptic input onto the excitatory population, the pyramidal cells are always activated before interneurons. It has been recently proposed in Chizhov et al. (2017) that the interneuron population should receive background synaptic input, which would allow the reproduction of interictal discharges in neural mass models.

Pre-ictal discharges are generated before seizure initiation and in the absence of inhibition when seizures have been established in the slice (Huberfeld et al., 2011). These oscillations are still generated in the absence of inhibitory population (Fig. 2C). Using the model, we show that in this case the background synaptic input to the excitatory population $I_{E}(t)$ is necessary to generate the periodic pre-ictal oscillations. When $I_{E}(t)$ is absent, there are no pre-ictal discharges in the model (Fig. $3 C$ ). We speculate that before seizure initiation the interneurons are becoming non-functional because of depolarization block (Ziburkus et al., 2006) and GABA $_{A}$ reversal (Lillis et al., 2012), thus allowing the pre-ictal discharges to be generated before seizure initiation (Huberfeld et al., 2011). The proposed model could explain the presence of preictal discharges only in the complete absence of inhibition (Fig. 2C). The possibility of pre-ictal discharge generation before seizure due to non-functional inhibition could be investigated in the future studies.

During seizures or ictal discharges, the frequency content of spiking activity might substantially change (Fig. $3 E$ ). This can be explained using the current model as due to the gradual increase of recurrent excitation $g_{E E}$ (Fig. $3 A$ ) or the increase of recurrent inhibition $g_{\|}$. Note that the frequency content of seizure oscillations in the end of it might be similar to the pre-ictal discharges (Figs. 2C, 3E). However, pre-ictal oscillations are possible in the model 
only in the absence of inhibition (Fig. 2C), as in the experimental data when the $\mathrm{GABA}_{\mathrm{A}}$ synaptic activity is completely blocked.

The primary advantage of our model compared to more abstract ones such as Jirsa et al. (2014) is that it provides more firm biophysical explanations linking single neuron properties to population dynamics (Johannesma, 1968; Gerstner and Kistler, 2002; Chizhov and Graham, 2007). Our approach could be extended to take into account the shunting effect of inhibition by adjusting the firing rate transfer function (Chizhov et al., 2014). To describe the additional mechanisms of seizure transition, the present model could include slow activity-dependent parameter changes similar to (Cressman et al., 2009; Ullah et al., 2009; Proix et al., 2014; Chizhov et al., 2018). There are multiple biophysical mechanisms that could play the role of slow variable bringing the network toward seizure (Naze et al., 2015), including dynamic ion concentration of extracellular potassium (Bazhenov et al., 2004), intracellular chloride (Jedlicka et al., 2011; Buchin et al., 2016b), and intracellular sodium (Krishnan and Bazhenov, 2011; Karus et al., 2015), in pyramidal cells. The population model could be further modified to incorporate these slow mechanisms to describe seizure initiation.

A common problem with neural mass models in general is their limited ability to generate the experimentally measurable signals (Lytton, 2008). In this work, we used the average voltage of the excitatory neural population as the approximation of the LFP signal near the neurons' somas (Ratnadurai-Giridharan et al., 2014). Given the distant dependence of the LFP signal, the current model should be considered only as a first approximation (Buzsáki et al., 2012). More detailed approaches describing populations of two-compartmental neurons (Chizhov, 2014; Chizhov et al., 2015) could also provide better approximation for the LFP.

Epilepsy is a complex phenomenon involving the dynamic interactions between multiple components of the nervous system (Lytton, 2008). In this work, we have investigated the particular role of inhibition and adaptation and their implications for seizure dynamics. Reconciling modeling results with experimental data, we have shown that seizure activity cannot be generated in the complete absence of the inhibitory population and adaption in the excitatory population. Further development of theoretical and experimental approaches in epilepsy research may lead to a better understanding of its mechanisms and the development of new therapeutic targets.

\section{References}

Bazhenov M, Timofeev I, Steriade M, Sejnowski TJ (2004) Potassium model for slow $(2-3 \mathrm{~Hz})$ in vivo neocortical paroxysmal oscillations. J Neurophysiol 92:1116-1132. CrossRef Medline

Beghi E, Berg A, Carpio A, Forsgren L, Hesdorffer DC, Hauser WA, Malmgren K, SS, Temkin N, Thurman D, Tomson T (2005) Comment on epileptic seizures and epilepsy: definitions proposed by the International League Against Epilepsy (ILAE) and the International Bureau for Epilepsy (IBE). Epilep 46:1698-1699. CrossRef

Brunel N, Wang XJ (2001) Effects of neuromodulation in a cortical network model of object working memory dominated by recurrent inhibition. J Comput Neurosci 11:63-85. [CrossRef]
Buchin A, Chizhov AV (2010a) Modified firing-rate model reproduces synchronization of a neuronal population receiving complex input. Opt Mem Neural Netw 19:166-171. CrossRef

Buchin A, Chizhov A (2010b) Firing-rate model of a population of adaptive neurons. Biofizika 55:592-599. CrossRef

Buchin A, Rieubland S, Häusser M, Gutkin BS, Roth A (2016a) Inverse stochastic resonance in cerebellar purkinje cells. PLoS Comput Biol 12:e1005000. CrossRef Medline

Buchin A, Chizhov A, Huberfeld G, Miles R, Gutkin BS (2016b) Reduced efficacy of the KCC2 cotransporter promotes epileptic oscillations in a subiculum network model. J Neurosci 36:1161911633. CrossRef

Buzsáki G, Anastassiou CA, Koch C (2012) The origin of extracellular fields and currents - EEG, ECoG, LFP and spikes. Nat Rev Neurosci 13:407-420. CrossRef Medline

Chin JH, Vora N (2014) The global burden of neurologic diseases. Neurology 83:349-351. CrossRef Medline

Chizhov AV (2002) Model of evoked activity of populations of neurons in the hippocampus. Biofizika 47:1007-1015.

Chizhov AV, Graham L (2007) Population model of hippocampal pyramidal neurons, linking a refractory density approach to conductance-based neurons. Phys Rev E Stat Nonlin Soft Matter Phys 75:011924. CrossRef

Chizhov AV, Rodrigues S, Terry JR (2007) A comparative analysis of an EEG model and a conductance-based neural population model. Phys Let A 369:31-36. CrossRef

Chizhov AV, Graham LJ (2008) Efficient evaluation of neuron populations receiving colored-noise current based on a refractory density method. Phys Rev E Stat Nonlin Soft Matter Phys 77:011910. CrossRef

Chizhov AV (2014) Conductance-based refractory density model of primary visual cortex. J Comput Neurosci 36:297-319. CrossRef

Chizhov AV, Sanchez-Aguilera A, Rodrigues S, de la Prida LM (2015) Simplest relationship between local field potential and intracellular signals in layered neural tissue. Phys Rev E Stat Nonlin Soft Matter Phys 92:062704. CrossRef

Chizhov AV, Amakhin DV, Zaitsev AV (2017) Computational model of interictal discharges triggered by interneurons. PLoS One 12: 0185752. CrossRef

Chizhov AV, Zefirov AV, Amakhin DV, Smirnova EY, Zaitsev AV (2018) Minimal model of interictal and ictal discharges "Epileptor2." PLoS Comput Biol 14:e1006186. CrossRef Medline

Cohen I, Navarro V, Clemenceau S, Baulac M, Miles R (2002) On the origin of interictal activity in human temporal lobe epilepsy in vitro. Science 298:1418-1421. CrossRef Medline

Cressman JR Jr, Ullah G, Ziburkus J, Schiff SJ, Barreto E (2009) The influence of sodium and potassium dynamics on excitability, seizures, and the stability of persistent states: I. Single neuron dynamics. J Comput Neurosci 26:159-170. CrossRef

de la Prida LM, Huberfeld G, Cohen I, Miles R (2006) Threshold behavior in the initiation of hippocampal population bursts. Neuron 49:131-142. CrossRef Medline

Demont-Guignard S, Benquet P, Gerber U, Wendling F (2009) Analysis of intracerebral EEG recordings of epileptic spikes: insights from a neural network model. IEEE Trans Biomed Eng 56:27822795. CrossRef Medline

Dietzel I, Heinemann U (1986) Dynamic variations of the brain cell microenvironment in relation to neuronal hyperactivity. Ann NY Acad Sci 481:72-84. Medline

Engel J, Bragin A, Staba R, Mody I (2009) High-frequency oscillations: what is normal and what is not? Epilepsia 50:598-604. CrossRef Medline

Florence G, Dahlem MA, Almeida ACG, Bassani JWM, Kurths J (2009) The role of extracellular potassium dynamics in the different stages of ictal bursting and spreading depression: a computational study. J Theor Biol 258:219-228. CrossRef

Fröhlich F, Bazhenov M (2006) Coexistence of tonic firing and bursting in cortical neurons. Phys Rev E Stat Nonlin Soft Matter Phys 74:031922. CrossRef 
Fröhlich F, Bazhenov M, Iragui-Madoz V, Sejnowski TJ (2008) Potassium dynamics in the epileptic cortex: new insights on an old topic. Neuroscientist 14:422-433. CrossRef Medline

Gerstner W, Kistler W (2002) Spiking neuron models: single neurons, populations, plasticity. Cambridge, UK: Cambridge University Press.

Hall D, Kuhlmann D (2013) Mechanisms of seizure propagation in 2-dimensional centre-surround recurrent networks. PLoS One 8:e71369. CrossRef

Huberfeld G, Wittner L, Clemenceau S, Baulac M, Kaila K, Miles R, Rivera C (2007) Perturbed chloride homeostasis and GABAergic signaling in human temporal lobe epilepsy. J Neurosci 27:98669873. CrossRef Medline

Huberfeld G, de la Prida ML, Pallud J, Cohen I, Le Van Quyen M, Adam C, Clemenceau S, Baulac M, Miles R (2011) Glutamatergic pre-ictal discharges emerge at the transition to seizure in human epilepsy. Nat Neurosci 14:627-634. CrossRef Medline

Jansen BH, Rit VG (1995) Electroencephalogram and visual evoked potential generation in a mathematical model of coupled cortical columns. Biol Cybern 73:357-366. CrossRef

Jedlicka P, Deller T, Gutkin BS, Backus KH (2011) Activity-dependent intracellular chloride accumulation and diffusion controls $\mathrm{GABA}(\mathrm{A})$ receptor-mediated synaptic transmission. Hippocampus 21:885-898.

Jensen MS, Azouz R, Yaari Y (1994) Variant firing patterns in rat hippocampal pyramidal cells modulated by extracellular potassium. J Neurophysiol 71:831-839. CrossRef

Jirsa V, Sporns O, Breakspear M, Deco G, Mclntosh AR (2010) Towards the virtual brain: network modeling of the intact and the damaged brain. Arch Ital de Biol 148(3):189-205. Medline

Jirsa VK, Stacey WC, Quilichini PP, Ivanov Al, Bernard C (2014) On the nature of seizure dynamics. Brain 137:2210-2230. CrossRef Medline

Johannesma PIM (1968) Diffusion models for the stochastic activity of neurons. In: Neural Networks, pp 116-144. Berlin: Springer Verlag.

Jung HY, Staff NP, Spruston N (2001) Action potential bursting in subicular pyramidal neurons is driven by a calcium tail current. $\mathrm{J}$ Neurosci 21:3312-3321. Medline

Kager H, Wadman WJ, Somjen GG (2000) Simulated seizures and spreading depression in a neuron model incorporating interstitial space and ion concentrations. J Neurophysiol 84:495-512. CrossRef

Karnup S, Stelzer A (1999) Temporal overlap of excitatory and inhibitory afferent input in guinea-pig CA1 pyramidal cells. J Physiol 516:485-504. CrossRef

Karus C, Mondragão MA, Ziemens D, Rose CR (2015) Astrocytes restrict discharge duration and neuronal sodium loads during recurrent network activity. Glia 63:936-957. CrossRef Medline

Krishnan GP, Bazhenov M (2011) lonic dynamics mediate spontaneous termination of seizures and postictal depression state. J Neurosci 31:8870-8882. CrossRef

Lillis KP, Kramer MA, Mertz J, Staley KJ, White JA (2012) Pyramidal cells accumulate chloride at seizure onset. Neurobiol Dis 47:358366. CrossRef Medline

Lytton WW (2008) Computer modelling of epilepsy. Nat Rev Neurosci 9:626-637. CrossRef Medline

Marder E, Taylor AL (2011) Multiple models to capture the variability in biological neurons and networks. Nat Neurosci 14:133-138. CrossRef Medline

Molaee-Ardekani B, Benquet P, Bartolomei F, Wendling F (2010) Computational modeling of high-frequency oscillations at the onset of neocortical partial seizures: from "altered structure" to "dysfunction." Neuroimage 52:1109-1122. CrossRef

Naze S, Bernard C, Jirsa V (2015) Computational modeling of seizure dynamics using coupled neuronal networks: factors shaping epileptiform activity. PLoS Comput Biol 11:e1004209. CrossRef

Pallud J, Le van Quyen M, Bielle F, Pellegrino C, Varlet P, Labussiere M, Cresto N, Baulac M, Duyckaerts C, Kourdougli N, Chazal G, Devaux B, Rivera C, Miles R, Capelle L, Huberfeld G (2014) Cortical GABAergic excitation contributes to epileptic activities around human glioma. Sci Transl Med 6:244ra89. CrossRef
Pinsky PF, Rinzel J (1994) Intrinsic and network rhythmogenesis in a reduced Traub model for CA3 neurons. J Comput Neurosci 1:3960. CrossRef

Prinz A, Bucher D, Marder E (2004) Similar network activity from disparate circuit parameters. Nat Neurosci 7:1345-1352. CrossRef Medline

Proix T, Bartolomei F, Chauvel P, Bernard C, Jirsa VK (2014) Permittivity coupling across brain regions determines seizure recruitment in partial epilepsy. J Neurosci 34:15009-15021. CrossRef Medline

Quilichini PP, Le Van Quyen M, Ivanov A, Turner DA, Carabalona A, Gozlan H, Esclapez M, Bernard C (2012) Hub GABA neurons mediate gamma-frequency oscillations at ictal-like event onset in the immature hippocampus. Neuron 74:57-64. CrossRef Medline Ratnadurai-Giridharan S, Stefanescu RA, Khargonekar PP, Carney PR, Talathi SS (2014) Genesis of interictal spikes in the CA1: a computational investigation. Front Neural Circuits 8:2. CrossRef

Renart A, Brunel N, Wang XJ (2004) Mean field theory of irregularly spiking neuronal populations and working memory in recurrent cortical networks. In: Computational neuroscience: a comprehensive approach, pp 425-463. Boca Raton, FL: CRC Press.

Rothstein JD, Patel S, Regan MR, Haenggeli C, Huang YH, Bergles DE, Jin L, Hoberg MD, Vidensky S, Chung DS, Toan SV, Bruijn LI, Su ZZ, Gupta P, Fisher PB (2005) Beta-lactam antibiotics offer neuroprotection by increasing glutamate transporter expression. Nature 433:73-77. CrossRef

Izhikevich EM (2007) Dynamical systems in neuroscience. Cambridge, MA: MIT Press.

Sivakumaran S, Cardarelli RA, Maguire J, Kelley MR, Silayeva L, Morrow DH, Mukherjee J, Moore YE, Mather RJ, Duggan ME, Brandon NJ, Dunlop J, Zicha S, Moss SJ, Deeb TZ (2015) Selective inhibition of KCC2 leads to hyperexcitability and epileptiform discharges in hippocampal slices and in vivo. J Neurosci 35:82918296. CrossRef Medline

Stiefel KM, Gutkin BS, Sejnowski TJ (2008) Cholinergic neuromodulation changes phase response curve shape and type in cortical pyramidal neurons. PLoS One 3:e3947. CrossRef Medline

Thomson DJ (1982) Spectrum estimation and harmonic analysis. Proc IEEE 70:1055-1096. CrossRef

Touboul J, Wendling F, Chauvel P, Faugeras O (2011) Neural mass activity, bifurcations, and epilepsy. Neural Comput 23:3232-3286. CrossRef Medline

Traub RD, Contreras D, Whittington MA (2005) Combined experimental/simulation studies of cellular and network mechanisms of epileptogenesis in vitro and in vivo. J Clin Neurophysiol 22:330342.

Ullah G, Cressman JF Jr, Barreto E, Schiff JS (2009) The influence of sodium and potassium dynamics on excitability, seizures, and the stability of persistent states: II. Network and glial dynamics. J Comput Neurosci 26:171-183. CrossRef

Ursino M, la Cara GE (2006) Travelling waves and EEG patterns during epileptic seizure: analysis with an integrate-and-fire neural network. J Theor Biol 242:171-187. CrossRef Medline

Wendling F, Bartolomei F, Bellanger JJ, Chauvel P (2002) Epileptic fast activity can be explained by a model of impaired GABAergic dendritic inhibition. Eur J Neurosci 15:1499-1508. CrossRef

Wendling F, Bartolomei F, Mina F, Huneau C, Benquet P (2012) Interictal spikes, fast ripples and seizures in partial epilepsiescombining multi-level computational models with experimental data. Eur J Neurosci 36:2164-2177. CrossRef Medline

Wilson HR, Cowan JD (1972) Excitatory and inhibitory interactions in localized populations of model neurons. Biophys J 12:1-24. CrossRef

Xiong ZQ, Stringer JL (1999) Astrocytic regulation of the recovery of extracellular potassium after seizures in vivo. Eur $\mathrm{J}$ Neurosci 11: 1677-1684. Medline

Ziburkus J, Cressman JR, Barreto E, Schiff SJ (2006) Interneuron and pyramidal cell interplay during in vitro seizure-like events. J Neurophysiol 95:3948-3954. CrossRef Medline 